OAK RIDGE NATIONAL LABORATORY

\section{MARTIN MARIETTA}

\section{MANAGED BY}

MARTIN MARIETTA ENERGY SYSTEMS, INC. FOR THE UNITED STATES DEPARTMENT OF ENERGY

\title{
An Updated Global Grid Point Surface Air Temperature Anomaly Data Set: 1851-1990
}
P. D. Jones
S. C. B. Raper
T. M. L. Wigley
B. S. G. Cherry
C. M. Goodess
B. Santer
P. M. Kelly
R. S. Bradley

H. F. Diaz

Environmental Sciences Division

Publication No. 3520 